Original Article

\title{
THE EFFECTS OF PRESBYOPIA ON PERFORMANCE VISION
}

Derek Cunningham ${ }^{1}$, Keith Smithson ${ }^{2}$, Amanda Nansay ${ }^{3}$, Fraser Horn ${ }^{4}$, Logan Kaleta ${ }^{5}$

${ }^{1}$ Dell Laser Consultants; dcunningham@sportsvisionpros.com; ${ }^{2}$ Northern Virginia Doctors of Optometry; ${ }^{3}$ Florida Institute of Sports Vision; ${ }^{4}$ Pacific University College of Optometry in Forest Grove, Oregon; ${ }^{5}$ Holy Cross Sports Medicine

Received: 17 May 2021; Accepted after revision: 13 June 2021; Published: 6 July 2021 .

*Author for correspondence:dcunningham@sportsvisionpros.com

\begin{abstract}
Presbyopia has been a condition that eye doctors have largely diagnosed based on age or clinical testing. Although the effects of presbyopia are typically very predictable in a clinical setting; its effects on real-world visual performance are far less predictable and relatively poorly understood. With more and more Baby Boomers and Gen X maintaining an active lifestyle, performance vision becomes a priority. This article aims to explore what is known about how visual performance can be maximized despite the progression of presbyopia and identifies areas where research is needed.
\end{abstract}

Keywords: presbyopia, visual performance,

\section{INTRODUCTION}

The most widely accepted model of presbyopic change in accommodation comes from the German physicist Hermann von Helmholtz. His theory was proposed over a century ago and has stood the test of time. He suggested that through ciliary muscle contraction, lens retention zonules would be relaxed and cause the pliable crystalline lens to thicken and increase curvature, mostly at the back of the lens. This resulted in increased focal power and allowed for nearer objects to remain in focus.

With age, the ability for the mechanical change in focus encounters significant resistance. This is due to the progressive stiffening of the crystalline lens. For most individuals, the change in focusing ability becomes functionally significant when the lens is not pliable enough to adjust focus from optical infinity to conventional reading distance (roughly $40 \mathrm{~cm}$ ). The change in the crystalline lens is remarkably reliable with age and the vast majority of patients will begin to encounter reading $(40 \mathrm{~cm})$ issues around the age of 45 .

Eye care has centered on the functional change of presbyopia and, as a result, it is highly conditioned to look for this age-specific change and provide a clinically measurable solution. If we solely look at stationary reading ability in the exam room, providing specific and reliable accommodative solutions to patients has been an accurate and highly successful method of dealing with presbyopia.

What happens to the presbyopes' visual system when they leave the stationary reading task is far less 
understood. Individuals must deal with a multitude of visual challenges that are not typically measured in the doctor's office. These visual demands are endless and include, but are not limited to: varying light levels, varying contrast levels, saccades, vergence, accommodative facility, color interpretation and more. The ability to process visual information with a decrease in accommodative ability can affect both cognitive understanding of an image and the neuromuscular response. These limitations may be minimally concerning for routine visual tasks, but can be limiting when the visual system is stressed. Add to that the number of Baby Boomers and Gen X who are committed to an active lifestyle, and performance vision in presbyopia reaches a critical mass.

Here, we will review what is known about the presbyopic effects on performance vision. Leading performance vision experts will detail how they maximize visual performance in presbyopia and suggest areas of research that are needed to manage the limitations of presbyopic vision in the future.

\section{THE HUMAN EXPERIENCE}

Patients may report that their vision changed "overnight"; however, eye doctors know that this is a process that starts at a very young age. As the accommodative ability decreases over time, a patient will notice a progression in the amount and severity of symptoms. This often starts off as some mild blur at near, especially when tired or with poor lighting. They may note that they have to increase the size of the text on their phone or use a light to read a menu in a dimly lit restaurant. The reduction in accommodation progresses and starts to induce more sustained blur for their near tasks. They may also see an impact on their medium range visual tasks, such as working on a computer.

In order to combat the blur, patients will hold objects further away or may purchase over-the-counter reading glasses to help with these changes. Without these compensatory measures, the patient's visual system often works harder to make the near object clear. This extra work often causes headaches over one's forehead and can also result in pain around the eyes. All of this visual-related pain is referred to as asthenopia. This can be a nuisance, initially, and then can become significant enough to impact one's work and ability to perform tasks accurately.

When an individual looks at a near target, their eyes aim at the near target (convergence) due to the coordination of their extra-ocular muscles (EOMs) to "aim" the eyes at the correct place. They will also increase their focusing (accommodation) for the distance where the target is at. In addition, one's pupils will constrict when they go from a distance to a near target. This interrelationship of the pupils, accommodation, and EOMs is known as the "near triad." During presbyopia, the accommodation ability is decreased; however, the signal to the EOMs and pupils remain. For some going through presbyopia, they can experience double vision, or diplopia, when looking at near due to the mismatch on accommodation and convergence ability. The near triad is also the reason better lighting, and the subsequent smaller pupil size, enhances near vision.

By the age of 50, individuals have an objective accommodation near zero; however, the subjective accommodation may be higher due to the depth of focus from the pupils. ${ }^{1}$ This subjective amount of accommodation is critical for visual performance. Most individuals will have sought relief by going to their eye doctor and receiving a prescription for glasses and/ or contact lenses to help compensate for presbyopia.

Most eye doctors' discussions regarding presbyopia focus on those tasks that are at a near range - reading, writing, use of smartphones or tablets, use of laptops, crossword puzzles, and more. These are, in general, in front of the individual and stationary. We do not often ask about the dynamics of how presbyopia impacts our daily function that is not directly in front of us. In addition, we do not ask about how presbyopia affects quality of life. We know that the individuals can have physical symptoms before being properly corrected, but does this solve everything? In 2003, McDonell found that when assessing for quality of life (QOL) indicators related to vision in presbyopes vs. non-presbyopes, those with presbyopia had worse QOL scores. ${ }^{2}$

When accommodative facility or amplitude decrease to the point it affects visual performance, this is presbyopia. This is not finite to age, gender, or geography, but a functional limitation that can be 
more debilitating to something that is purely based on the demands that the visual system are put under. Here the article tries to detail the progressing limits of presbyopia and how they limit performance.

\section{UNDERSTANDING OF FUNCTIONAL LIMITATIONS OF PRESBYOPIA}

Because the crystalline lens stiffens slowly and progressively in most cases, there is likely a significant adaptation that the subject experiences. These adaptation responses are often behavioral such as avoiding near visual focus, better anticipation of moving objects, or adjusting position/environment to keep target at a more functional distance.

When the visual system is used to its limits, the deficiencies can be evaluated in an isolated manner. For much of our critical analysis of the presbyopic visual system, it may be difficult to separate the visual systems specific limitations from general aging changes that could be considered neurological or neuromuscular in nature. Because these possible changes happen simultaneously, that distinction will not be addressed at this time.

\section{BEYOND CLASSIC NEAR BLUR}

\section{Contrast sensitivity}

Contrast sensitivity can often be thought of as a more practical measure of vision quality than even visual acuity. Visual acuity charts measure vision under a very controlled situation with one hundred percent black on white contrast. Although this is a very repeatable method of measuring one aspect of the visual system, it is rarely encountered in the real world. Real world vision is a mixture of highly variable contrast levels seen under different lighting conditions.

A study in 2013 looked at the effects of aging on contrast sensitivity. The study found that contrast sensitivity starts to decline at the same time that presbyopia becomes an issue. ${ }^{3}$ To give a related functional perspective, a recent study showed that alcohol consumption also temporarily decreases contrast sensitivity. ${ }^{4}$

\section{Reaction times/accommodative facility}

Age has been associated with diminished reaction time due to multiple factors. However, reduction in speed of accommodation may be a significant factor in this deterioration if the subject needs to look at targets at multiple distances. As the natural crystalline lens hardens throughout a patient's lifetime, their speed of focus change decreases. A study out of the United Kingdom showed that simple reaction times show little slowing until around age 50, whereas choice reaction times slow throughout the adult range. ${ }^{5}$ This study would suggest that presbyopia could be a key contributor to delayed reaction times.

\section{Decreasing amplitude}

The range in which an object can be kept in clear focus has been well studied in the evolving presbyope. The rate at which it reduces is not only genetically predetermined but also can be further deteriorated by stress placed on the visual system. One study showed that the total cumulative near-work time was negatively correlated with accommodative facility and positively correlated with the number of asthenopic symptoms. ${ }^{6}$ Furthermore, significant correlations were found between total near-work time and blurred vision, and blurred vision and reduced accommodative facility.

\section{Color perception change (with cataract formation)}

Two interesting studies suggest that the presbyope that stresses their visual and physical system may have measurable color perception effects compared with that of younger norms. One study showed that intense physical effort affects color vision with the tritan axis being predominantly affected (blue-yellow). ${ }^{7}$ Another study showed that the natural aging changes in the human lens diminish color sensitivity for all three cone classes, with the greatest decrease for S-wavelength sensitive cones (blue-yellow). ${ }^{8}$

\section{Eye alignment}

As mentioned earlier, presbyopes undergo progressive stresses on their eye alignment as focus is reduced. It was revealed that $87 \%$ of the presbyopic patients had binocularity shortage. Further, the reduction of binocular field area in extreme grade was seen in $6 \%$ of cases. $^{9}$

\section{Saccades}

Saccades are the eyes' ability to move from one focal point in space to another. This is done through a coordinated change in the eye muscles. It has been 
shown that age, stimulus intensity, and eccentricity had a statistically significant effect on saccade reaction time, but gender does not. ${ }^{10}$ Using the Righteye system (patients sitting $60 \mathrm{~cm}$ away from screen), one study showed that latency and accuracy of saccades start to decrease in the 29-52 year old age group, what may be considered early presbyopia. ${ }^{11}$

\section{Glare recovery}

Our ability to maintain focus or recover vision in the presence of glare seems to diminish with age. This has significant implications in both daily tasks and high level visual performance. From oncoming bright headlights at night to trying to fixate on a moving target that is passing a light source, the visual system progressively struggles as people age. Frank Schieber from the University of South Dakota Department of Psychology was able to demonstrate significantly longer glare recovery times with 40-55-year-old subjects when compared with that of 18-24 year old subjects. ${ }^{12}$

It becomes clear that there are numerous mechanisms that begin to limit vision performance beyond visual acuity as the eyes start to suffer from presbyopia.

\section{ASPECTS OF VISION THAT CAN BE IMPROVED}

Eye care providers will often be the first to explain the cause of the frustrating changes of presbyopia, but most importantly they will discuss the ways to help the patient cope with those changes. Although the patient may never be able to have the "same" vision they once did, new technology has given us options to compensate for changes to the accommodative system. For sports and active occupations in presbyopes, various options will be explored, from the more traditional and atypical spectacle options to multi-focal contact lenses. New innovations in miotics and surgical correction will lead to necessary education of patients, as well as providers, to assure everyone knows the correction options for presbyopic management.

\section{GLASSES}

When discussing eyeglasses for presbyopia, the main discussion points are whether the solution can be a singular lens with multifocus solution, or multiple lenses with singular focus solution. The limitations involved with singular focus tools are that each of these tools would be appropriate for focus only at one specific distance. For example, a "computer lens" could be set with a $66-\mathrm{cm}$ focal point, whereas a lens intended for use with reading a book or phone may be set and manufactured with a $40-\mathrm{cm}$ focal point. Multifocal lens options, commonly known as progressive addition lenses or PALs, are intended to be a multi-use tool with a variable focus at all ranges from optical infinity ( 6 meters or beyond) and all the way up to a near vision focal point, usually $40 \mathrm{~cm}$, and incorporating all fields of view in between those two extremes.

The benefit of this lens technology is that all targets being viewed from any distance can be seen clearly if the viewer is angled properly to access the correct portion of the PAL. However, head movements are often required to find the correct focal points. Consequently, head position could create a compromised posture in sports like target shooting or golf. As an example, a golfer standing over a putt and looking in down gaze would be viewing a mid-range target (the ball) through the near vision portion of the lens (the extreme bottom of the lens) unless they drop their chin and attempt to view the ball with a mid-portion of their PAL. However, with training and adaptation, these skills can still be managed with most PALs. A second consideration when using PALs for dynamic activities, is the peripheral vision distortion that can be inherent optically in the physical design of some PALs. PALs are designed and cut like an hourglass with the most precise vision in a vertical column in the middle of the lens. It is common for targets in the periphery to be blurred. Many new wearers are instructed saying "point your nose at what you are trying to look at" to position this column of best visual clarity straight ahead for best results. In dynamic environments, peripheral vision and peripheral awareness can be critical, such as in contact sports such as basketball, football or soccer, or in motor sports such as motocross or racing.

The final consideration regarding use of PALs in dynamic environments is the limited viewing area allocated for each prescription increment. The vertical heights of all PALs are delineated to include all incremental prescriptions that a patient would need between their distance refractive correction and their 
near point correction. With advancing age, more increments are added to that vertical column of vision. The effect of this is a smaller viewing window for each prescription and less overall "real estate" for that patient to utilize when viewing a dynamic target at a specific distance. As an alternative to PALs, segmented bifocals or trifocals could be used for presbyopes. The benefit to these lenses are larger viewing areas in the two or three segments included in the lens and less peripheral vision impact. The ability to use occupational segments, such as an inverted bifocal for near viewing needed above an athlete, such as a pilot or a shooter, is also possible. However, the limitation of the segmented lenses is in the more dramatic jump between powers and the lack of a smooth visual transition zone between multiple powers. The concern is that the image could "jump" from one power to another during eye tracking or scanning, or the target may lack clarity at a specific distance if the two or three segments do not specifically account for that viewing distance.

The peripheral distortion of PAL lenses seems to be more acceptable to presbyopic drivers than the power limitations of segmented bifocals. ${ }^{13}$ This is supported by overall spectacle sales and an Australian study that satisfaction was significantly higher for progressive spectacles than bifocals for driving. ${ }^{13}$ A still more telling study from London that assessed all spectacle forms of presbyopia correction showed that in all quality of vision (QoV) metrics, regardless of the far, intermediate or near blur assessment, QoV was rated higher by patients whose main tasks were far focused, than those who principally conduct intermediate tasks and worse still for those whose main tasks were near, regardless of the form of correction. ${ }^{14}$

\section{CONTACTS}

While contact lenses have become a staple for vision correction, many patients are still not aware of the newest options for presbyopic correction. The cause of this is likely due to a lack of education of what options would currently be available to meet the patients' needs. When fitting a presbyope in contacts, there are many aspects to consider. Providers need to find a lens that fits into the correct parameters (base curve, diameter, power) while choosing the modality of soft, gas permeable or hybrid options. A successful presbyopic contact lens fitting will take into account the lifestyle and the visual needs of the patient. A presbyope in the year 2021 is doing static visual tasks in distance and near environments. Many patients in this age group are spending many hours on a computer at various degrees of intermediate distances of using laptops, desk top monitors, as well as tablets. This age group, as previously discussed, is physically active and participates in various recreational activities and sports that will still necessitate aspects of performance vision that their younger non-presbyopic counterparts require.

Also of note, any patient that requires the most clear and consistent vision, will need to be in a contact lens that provides a stable and clear tear film. As patients age, they will be more likely to suffer from ocular surface changes that will require the best materials to last all day without causing discomfort or blurry vision. As previously discussed, presbyopes may already have a reduction of contrast sensitivity that can only be further impacted without choosing the best material.

\section{Distance only contacts with reading glasses as needed}

This is the most common correction eye care providers have given patients for years. It is an "easy" correction that takes doctors minimal chair time, and allows patients to simply put on a pair of reading glasses over their contacts when their focusing system isn't doing enough for their near visual needs. This will likely give patients the clearest long-distance, binocular vision, and less adaptation, especially for early stage presbyopes that only need assistance in their near vision $(40 \mathrm{~cm})$ and closer. The option can be done with any patient that was previously fit into contact lenses, without changing their modality of contact lens.

This option will leave some patients with the emotional/social baggage of "looking older" as well as inconvenience them with the need to keep glasses at hand for when they need them. For patients that are more advanced in their presbyopia, there will be a point where intermediate distance needs and near vision needs will no longer be able to be corrected 
with only two powers. This will necessitate a change in visual solution.

Athletes that are early presbyopes may not feel the obvious burden of accommodative changes unless their near visual system is stressed. Exceptions may be the golfer that needs to see a score card or a shooter that needs to see a sight/reticle at close range. While a golfer can easily put on readers to see a score card, a shooter will not have the same option of using readers as easily.

\section{MONOVISION}

Monovision is the term used for visual correction that leaves one eye (typically the dominant eye) clear in the distance, while the other eye is corrected for near vision. This is a visual solution that is most popular in contact lens and LASIK correction only, due to image size disparity that this type of correction in glasses often creates. This visual solution is also straightforward for the provider, allowing flexibility in modalities as well as the correction of astigmatic refractive errors fairly easily.

This option will require one very important trait for the patient: suppression of the eye that is not clear at whatever viewing distance they are looking at during any particular moment. There is not usually much of a "grey" area with these patients. They will either easily adapt to monovision, or they will not. Considering the visual tasks of today's world that not only rely heavily on intermediate tasks, there is always the possibility that a patient will do fine in office, looking at a distance and near chart, but not adapt in their day-to-day visual requirements.

Depth perception restrictions with this type of visual correction is not only a problem for our athletes, but also for anyone that drives a car. Many people will do fine using other localization cues, but changes in the way depth is perceived should always be discussed for patients to consider.

An exception to the monovision rule of "dominant eye in distance correction" will be seen in one sportshooting. Many professional and recreational shooters will prefer to have a clear site with their dominant eye. The center of a clear target is the same as the center of a blurry target, but if a site can't be seen, they won't be able to properly align their weapon.

\section{MULTIFOCALS/ MODIFIED MULTIFOCAL/ MF TORICS}

In this type of contact lens correction, both distance and near vision can be obtained binocularly. If done well, this could be the closest to a pre-presbyopic lifestyle.

The downside to this seemingly perfect option is that many providers find their patients "compromise" in distance and/or near to have both. This could potentially be remedied by more education about how to successfully fit many of the new designs that have a higher rate of success

Contact lenses have historically been the vision correction of choice for athletes. A full and undistorted peripheral field of view along with no external facial devices are key benefits for this method or correction over others. Contact lenses also have the advantage of always being optically centered over the line of sight, maximizing vision in all angles of gaze compered to spectacles. Conversely, contact lenses can be difficult for patients to use. Patients need to overcome a natural aversion to touching their eyes and may have some significant discomfort.

\section{RLE}

Surgical correction of cataracts has provided an important area of research and innovation for presbyopia. Since cataract surgery is one of the most performed surgeries on earth and removal of the natural crystalline lens inherently creates presbyopia; this provides us with a massive base population to study the benefits of presbyopia. With advancements in lens replacement technology, we have seen a rapid evolution in artificial intraocular lenses that can provide varying levels of far and near vision without the use of spectacles or contact lenses.

Currently the two most popular forms of presbyopia correcting intraocular lenses are multifocals and extended depth of focus technologies. Although all of these lenses provide varying levels of near focus and quality, they all suffer light distortion effects that are inherent to light manipulation. Luckily these distortions are rarely noticed in well-lit situations, but they can be problematic for some in low light. This is most commonly seen when the subject is looking at a dark 
background with point sources of light. Common examples include night driving and looking at stars. The low-light glare and halos that are seen with these lenses are primarily due to the larger pupil aperture size in low light and sub threshold amounts of light. It is important to note that although these low light distortions are visible to most, few will feel that they are debilitating and in our clinical experience even less will report that they limit their quality of life.

The main advantages of an intraocular lens exchange for presbyopes include reduced reliance of near vision aids such as glasses and contacts, but also it stops the progressive vision change that the natural human lens will undergo as long as it is in the eye. A stable and predictable visual system is a major benefit for visual performance. Glasses and contacts produce their own optical distortions, but the external auxiliary nature of those corrections is their major limitation. Not having to put anything in your eye or on your face is a major asset in vision performance. The main disadvantages of this mode of correction include significant patient cost, risk associated with eye surgery, and potential for significant nighttime glare.

\section{NEURAL TRAINING}

Suggested Neural or visual training for presbyopes would include eye tracking and saccadic function training to attempt to allow athletes to more smoothly focus through multiple visual zones of a PAL. The assumption is that adaptation to PALs and specifically PALs for dynamic sport could be improved in function if a patient is more smoothly able to migrate through the various visual zones and seamlessly find clarity when needed symmetrically with both eyes.

Secondly, an area of potential research would be to train peripheral awareness. The goal of peripheral awareness training would be to allow the athlete to more completely process their peripheral targets with any optical limitations in place for the necessity of improving clarity.

Fusional vergence training could additionally be attempted to aid the presbyope in converging the eye muscles to view near targets and diverge effectively to accurately view far targets. These visual skills are required for the athlete/driver to achieve the most precise levels of three-dimensional depth perception.
Most PAL lenses require a functional convergence movement down and in when viewing near targets to allow the eyes to stay smoothly in the clarity column for the PAL. $15-20 \%$ of the general population exhibits under convergence which could limit success when moving the eye dynamically to view multiple targets through multiple powered lenses. ${ }^{15}$

It is assumed that training the above visual skills in a virtual or augmented reality environment could be beneficial, depth perception, peripheral awareness, eye tracking and scanning and visual processing speed could all be instituted in a simulated real space environment to maximize the effect of the training and create good transference potential to real world dynamic environment scenarios.

Due to limitations in human lens flexibility and ciliary muscle flexibility during presbyopia, accommodative function training would be expected to yield little benefit.

\section{MIOTICS}

There is a natural constriction of the pupil with age. This is thought to be a natural adaptation to increase depth of focus (trying to offset some of the reduction in accommodation). Constricting the pupil to increase the eyes' depth of focus has been well documented for decades. Pharmacological agents to constrict the pupil have been readily available and heavily studied for decades. The ability to temporarily restore significant near vision with simply putting a drop in the eye has always seemed equally attractive to both eye doctors and patients. The major limitation of this type of vision correction has been the medication side effects. Significant brow ache or headache is so common with this class of eye drops, that few can stand the symptoms. There are also significant patient variability and pharmacodynamic hurdles that lead to varying pupil sizes, so variable vision benefits.

Optimally, we would want a drop that would consistently produce a pupil size between $2-3 \mathrm{~mm}$. Too small and we get night vision issues and degradation of the image due to diffraction. Too large and we do not get enough near focus ability. A fixed 2-mm to 3-mm small pupil or a $30 \%$ pupil miosis can produce near visual acuity gains without significant losses to distance acuity or image quality, and therefore can 
be considered as optimal for a presbyope experiencing a wide range of light levels. ${ }^{16}$ This may not give perfect clarity at a very near working distance like 40 $\mathrm{cm}$, but more likely give very functional clarity for modern needs like phones and computers (a 50-75 cm distance target)

Recently we have seen several formulations of drops under review by the FDA that claim to do just this. The key to their success will be dependent on their ability to eliminate the brow/headache symptoms and if they can provide consistent near vision for a reasonable amount of time. If they are able to do this, we could have a product with all the vision performance benefits of a refractive lens exchange without the risk or cost. The major limitations of this mode of correction would include its temporary nature and possible inter patient variability.

This may be a very attractive option to presbyopic athletes as most of their vision needs are at an intermediate distance where this correction will excel. This will also allow athletes to self-administer the drop when needed and not deal with the constraints and limitations of glasses and contacts. From a vision performance standpoint, eliminating the peripheral distortion of spectacle lenses or the comfort and dryness issues with contact lenses, is an attractive choice

\section{EXPERIMENTAL RESEARCH}

There are several early studies on new technologies that address presbyopia by softening the human crystalline lens with drops or LASERs, but currently there is no long term data on their efficacy or side effects.

\section{PRACTICAL EXAMPLES WHERE PRESBYOPIA LIMITS VISUAL PERFORMANCE}

\section{Shooter-near sights}

Shooters pose an interesting problem when they approach presbyopia. A large majority of athletes have a high demand for clear distance vision. Occasionally, an intermediate need will be applicable for drivers or even the near vision demand of a golfer looking at a score card. Most shooters, however, will need to look through some sort of a sight or reticle. For a non-presbyope, looking from distance to near is a quick and effortless change. When the accommodative system begins to change for a presbyope, the eye care provider must decide the best way to compensate for the loss of flexibility in the focusing system by using one of several different options in several different modalities.

Some shooters may find progressive glasses or bifocals as an acceptable correction option, but a large majority will not. As discussed previously, a certain head posture must be observed to view through each portion of the lens. Depending on the type of weapon used (handgun vs shot gun) as well as the type of targets they use (static vs dynamic clays or game), their head posture as well as visual demands can negatively be influenced by a progressive design. This is why many shooters that wear glasses will opt for correction that would typically not be done, monovision.

Monovision will always be done in an Rx after one very important test: dominance. In any other situation, the dominant eye will be given the full distance correction, while the non-dominant eye will be put in either the full near correction or part of it. When we deal with shooting however, the athlete would often prefer to have their dominant eye on their site/ reticle. The fact that "the center of a blurry target is the same as the center of a clear target" is the reason why a shooter may prefer to have their dominant eye clearly focused on the site, and let the non-dominant eye be the one that watches the target.

Again, another problem may come into play when considering that not all shooters are same hand and eye dominant. Cross dominant shooters may have adapted to having their non-dominant eye looking through the sight. It could be proposed that those presbyopes may have a harder time adapting to viewing through a monovision prescription.

Considering contacts as a solution may give better options due to prescription that is not based on head posture. Monovision contact lenses would offer the same solution without the restrictions of head posture or a frame affecting field of view.

Multifocal contact lenses would likely be the best option for a shooter. This type of lens can give clear vision at all viewing distances as well as the option for binocularity. Again, a limitation of contacts is often that the vision is not perfect due to the aspheric rings that offer many different variable powers in one 
lens. Presbyopes are also most susceptible to dry eye that can easily degrade the quality of vision in their contacts if the proper fit and material is not selected.

One final component that can lead to a disruption of performance for a shooter that must now cope with a new accommodative correction lies in the fact that not all shooting sports allow the athlete to remain stationary. From rotating body positions to the necessity to run to different positions, these athletes will need to learn to compensate for the adaptation to new lenses in a dynamic environment. The "swim" of a progressive lens or the loss of depth perception in any type of monovision correction, can no doubt have a negative impact on performance.

\section{Motorcycle racer}

Daily driving is surprisingly demanding on the visual system, but luckily we have developed multiple protocols to ease the burden. These include a comfortable and static driving position, predictable positions and rules for other cars on the road, and limited speeds to allow for sufficient reaction times. Motorcycle racers enjoy none of the aforementioned luxuries. Their visual demands are among the most stressed in sport and this can be a breaking point for a presbyope trying to compete.

Elite motorcycle racers must hang off the side of their machines at extreme angles so their head is often only inches off the ground. They do not use primary gaze throughout the entire race and are almost always looking up through their brow or ahead, leaning forward with a tilted head. This highly abnormal and varied eye position will affect spatial awareness and response times. Both of these metrics suffer in even the most basic studies as we age into presbyopia. This is then compounded by highly unpredictable racers in close proximity while traveling at highly variable speeds (from $50 \mathrm{kph}$ to $320 \mathrm{kph}$ ). This adds more stress to susceptible visual skills such as saccades and multiple object tracking.

The most obvious visual requirement to suffer can also be the most difficult to deal with historically. Reduced accommodative ability and response time becomes very problematic for presbyopes who need to see their gages and then quickly refocus at distance. Not only is the ability to focus up close reduced, the speed at which this happens slows considerably. Reduction in the ability and speed to see up close is highly problematic because split section decisions are constantly needed for safe and effective travel. For example, when a racer is traveling at $320 \mathrm{kph}$, they are covering more than 89 meters per second. In our testing, a young racer will be able to change their focus from the distance down to read their gages and refocus in the distance in about 1.2 seconds. That means they are covering more than 106 meters without being able to react appropriately. The same testing showed that presbyopes can commonly take up to 3 seconds with appropriate eye wear, meaning that they are covering over 267 meters without the ability to react appropriately. That is an astonishing extra 161 meters without being able to react.

Progressive spectacle lenses are routinely worn for recreational driving and many athletes attempt to use them in a situation like this without knowing that this is a dangerous form of correction for these athletes for numerous reasons. The first is the basic space requirement for the spectacles to fit properly under the helmet. The second is that the vision portions and peripheral distortion of the lenses are optimized for primary gaze and require free head movement to target. This makes the lens detrimental and often dangerous to wear in this situation. There is also an issue of fogging or moisture accumulation on the lens during intense physical activity, dramatically reducing contrast. The last is related to the significant $\mathrm{G}$ forces the head is placed under during acceleration and breaking. These forces often cause eyewear to move or even fall off under stressed conditions.

Through the use of new technology multifocal contact lenses many of the racers have been able to maintain much of their competitive visual performance on track. The problems of older generation presbyopia contacts having issues with comfort and overall clarity of vision, are rarely now seen. Excellent clarity is now a norm at both near and far distances as well as speed of focus. We were able to test presbyopic racers in a full tuck position on their bikes to assess speed of distance to gauge to distance focus ability. With the newer generation multifocal lens we found the ability to focus on a near gauge at $30 \mathrm{~cm}$ from their face and back to distance in times that were much closer 
to younger norms. This is currently our correction of choice for presbyopic motorsport athletes.

\section{Daily driver}

Successfully and safely driving requires a multitude of visual skills being used together for maximum visual function. The driver needs clarity of vision at multiple ranges, distance to cars in traffic, to close range in dashboard viewing. They need quick and efficient eye tracking to track targets in motion, such as someone crossing the street or merging onto the road. The need to scan quickly between multiple targets such as multiple cars in traffic in oncoming and outgoing traffic. They need effective peripheral awareness to see cars to the side when changing lanes or merging into traffic. With the considerations listed above for optical solutions, maximizing visual skills around any potential limitations would be critical for best visual function.

A presbyopic driver will have altered eye and head movement with different types of presbyopic corrections. The longer path length of eye and head movements and greater number of saccades associated with the spectacle presbyopic corrections may affect some aspects of driving performance. This was shown in a study showed the path length of eye movements while viewing and responding to drivingrelated traffic scenes (participants were required to view videotape recordings of traffic scenes, track a reference vehicle, and identify a series of peripherally presented targets) was significantly longer when wearing bifocal and PAL spectacles than monovision and multifocal contact lenses. ${ }^{17}$

\section{AREAS OF RESEARCH NEEDED}

How the altering visual system in presbyopia affects musculoskeletal performance: Although we have extensive exam lane data on presbyopia, there is a sizable gap in our understanding of how the visual input changes can alter how the body responds.

New technologies in neural/visual training are beginning to provide us with unlimited options in assessing the visual systems abilities and limitations. Virtual reality and augmented reality have the instant ability to harvest data and provide reliable and precise training protocols. This pliability and precision come at the expense of isolating the visual system from many of the other systems involved in performance such as the musculoskeletal system. There still needs to be extensive research as to how applicable the virtual/augmented reality training will be to real world performance.

More definitive studies on the effects of presbyopia on driving. Driving simulators now have the ability to mimic multiple dynamic vision requirements. Due to the high speed movement and multiple object tracking required, driving simulators represent an excellent platform for study that can have significant benefits on our daily lives.

Adaptive optics - As new optical devices are developed that have either integrated displays or adaptive optics, it is unclear how the changing presbyopic visual system will perform.

Miotic agents and dynamic visual performance. Although this concept has been known for decades, it has yet to be studied in detail due to lack of widespread use.

\section{RECOMMENDATIONS FOR VISUAL PERFORMANCE IN PRESBYOPES}

We can see that the visual system in a presbyope undergoes numerous functional limitations. We are seeing an increasing number of presbyopes perform at a very high level, and these visual demands become increasingly more limiting as the athlete ages. There are learned behaviors that can compensate for some of these limitations (such as anticipation or position modification), but they will inevitably limit performance as well.

\section{REDUCE DISTORTION}

If wearing vision correction aids such as contacts or glasses, optic distortion should be a key concern. Contact lenses will typically provide the best peripheral awareness and least peripheral distortion while attempting to aid in near vision. If spectacles are chosen, the need for more head movement should be addressed. It is also important to minimize optical aberrations with high clarity lenses and advanced lens coatings. With internal performance factors limitations beginning to manifest, presybyopes with 
performance vision needs cannot afford to deal with reduced quality optics as well.

\section{FREEDOM FROM AIDS}

Aiding focus ability in presbyopes without the need for vision aids and the restrictions that come with them is a very appealing concept. Currently this is available with surgically implanted intraocular lenses, but this has limited availability due to sizable cost and time commitment. With the impending release of presbyopia "aiding" eye drops, the cost, commitment and risk barriers will all come down. This will be a readily available option for almost every presbyope to experiment with enhanced focus ability. The pharmaceutical correction of presbyopia is especially important to athletes because it may eliminate the need for vision aids that can limit performance.

Although this is poised to be a very exciting development for vision performance with presbyopia, it is important to note that it is unknown how the specifics of dynamic vision will be affected by this therapy. To date we do not have studies or data that suggest how the use of these drops may affect metrics such as saccades, reaction time, glare, accommodative facility, and so on.

\section{Limit Glare}

The debilitating effects of glare become more problematic as the visual system ages. Not only is glare massively distracting for a presbyope, it can also cause the visual system to reset and temporarily stop all visual input. Distortion free quality tinted eyewear and shields continue to be the main treatment to mitigate the effect of glare. Various tints have advantages over others depending on ambient lighting levels as well as target color or even speed of movement.

There are evolving technologies that may provide presbyopes with glare mitigation while preserving vision quality. One technology currently available is a photochromic contact lens. This contact lens is able to transition to almost completely clear view in the absence of UV light but block 70\% of visible light at full UV activation. The new presbyopia "aid" eye drops may be very useful in reducing low light glare. Eye doctors have used this class of medications for years in order to reduce night glare and halo symptoms in patients. Further testing will be needed once commercially available formulations are available.

\section{CONCLUSION}

It is remarkable that so little is fully understood about a condition that will eventually handicap every person on earth who lives long enough. The magnitude of our knowledge on how presbyopia affects people in a static setting is in stark contrast to our ignorance on how presbyopia affects dynamic vision outside of the lab. Through collaborative research and advancements in technology, the effect of presbyopia on performance vision, and therefore ways to assist, are slowly coming into focus.

\section{REFERENCES}

1. Hamasaki D, Ong J, Marg E: The amplitude of accommodation in presbyopia. Am J optom Arch Am Acadm Optom 33:3-14, 1956

2. McDonnell PJ, Lee P, Spritzer K, Lindblad AS, Hays RD. Associations of Presbyopia With VisionTargeted Health-Related Quality of Life. Arch Ophthalmol. 2003;121(11):1577-1581. doi:10.1001/ archopht.121.11.1577

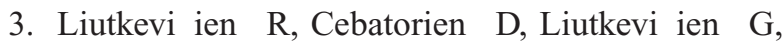
Jašinskas V, Zali囚nien囚 D. Associations between contrast sensitivity and aging. Medicina (Kaunas). 2013;49(6):273-7. PMID: 24248007.

4. Casares-López M, Castro-Torres JJ, Martino F, OrtizPeregrina S, Ortiz C, Anera RG. Contrast sensitivity and retinal straylight after alcohol consumption: effects on driving performance. Sci Rep. 2020 Aug 12;10(1):13599. doi: 10.1038/s41598-020-70645-3. PMID: 32788613; PMCID: PMC7423611.iho

5. Der G, Deary IJ. Age and sex differences in reaction time in adulthood: results from the United Kingdom Health and Lifestyle Survey. Psychol Aging. 2006 Mar;21(1):62-73. doi: 10.1037/0882-7974.21.1.62. Erratum in: Psychol Aging. 2009 Mar;24(1):229. PMID: 16594792.

6. Iribarren R, Fornaciari A, Hung GK. Effect of cumulative nearwork on accommodative facility and asthenopia. Int Ophthalmol. 2001;24(4):205-12. doi: 10.1023/a:1022521228541. PMID: 12678397.

7. Tekavcic B, Mili冈 R囚, Pompe MT. Does Physical Fatigue Affect Color Vision? Sports Med Int Open.

DOI:https://doi.org/10.22374/jspv.v3i1.10

J Sports Perf Vis 3(1):e18-e31; June 6, 2021.

This article is distributed under the terms of the Creative Commons Attribution-Non Commercial 4.0 International License. ( C Cunningham et al 
2017 Aug 2;1(5):E155-E159. doi: 10.1055/s-0043115378. PMID: 30539101; PMCID: PMC6226077.

8. Mehta U, Diep A, Nguyen K, Le B, Yuh C, Frambach C, Doan J, Wei A, Palma AM, Farid M, Garg S, Kedhar S, Wade M, Marshall KA, Jameson KA, Cristina Kenney M, Browne AW. Quantifying Color Vision Changes Associated With Cataracts Using Cone Contrast Thresholds. Transl Vis Sci Technol. 2020 Nov 3;9(12):11. doi: 10.1167/tvst.9.12.11. PMID: 33200052 ; PMCID: PMC7645251.

9. Rozanova OI, Shchuko AG, Mischenko TS. Fundamentals of Presbyopia: visual processing and binocularity in its transformation. Eye Vis (Lond). 2018 Jan 25;5:1. doi: 10.1186/s40662-018-0095-0. PMID: 29417087; PMCID: PMC5784700.

10. Mazumdar D, Meethal NSK, Panday M, Asokan R, Thepass G, George RJ, van der Steen J, Pel JJM. Effect of Age, Sex, Stimulus Intensity, and Eccentricity on Saccadic Reaction Time in Eye Movement Perimetry. Transl Vis Sci Technol. 2019 Jul 30;8(4):13. doi: 10.1167/tvst.8.4.13. PMID: 31388465; PMCID: PMC6670042.

11. Murray et al. An Examination of the Oculomotor Metrics within a Suite of Digitized Eye Tracking Tests. Vision Development and Rehabilitation. 2019 Dec. 5:4.

12. Schieber, F. Age and Glare Recovery Time for LowContrast Stimuli. Dep of Psychology. University of South Dakota. apps.usd.edu.
13. Chu, Byoung Sun M.Optom.; Wood, Joanne M. Ph.D.; Collins, Michael J. Ph.D. Effect of Presbyopic Vision Corrections on Perceptions of Driving Difficulty, Eye \& Contact Lens: Science \& Clinical Practice: May 2009 - Volume 35 - Issue 3 - p 133-143 doi: 10.1097/ ICL.0b013e3181a1435The impact of presbyopic spectacles and contact lenses on driving performance

14. Sivardeen A, McAlinden C, Wolffsohn JS. Presbyopic correction use and its impact on quality of vision symptoms. J Optom. 2020;13(1):29-34. doi:10.1016/j. optom.2018.12.004

15. Ghadban R, Martinez JM, Diehl NN, Mohney BG. The incidence and clinical characteristics of adultonset convergence insufficiency. Ophthalmology. 2015 May;122(5):1056-9. doi: 10.1016/j.ophtha.2014.12.010. Epub 2015 Jan 24. PMID: 25626756; PMCID: PMC4414695.

16. Xu R, Thibos L, Bradley A. Effect of Target Luminance on Optimum Pupil Diameter for Presbyopic Eyes. Optom Vis Sci. 2016 Nov;93(11):1409-1419. doi: 10.1097/OPX.0000000000000963. PMID: 27560851.

17. Chu, Byoung Sun; Wood, Joanne M.; Collins, Michael J. Influence of Presbyopic Corrections on Driving-Related Eye and Head Movements, Optometry and Vision Science: November 2009 - Volume 86 - Issue 11 - p E1267-E1275 doi: 10.1097/OPX.0b013e3181bb41f2htd 


\section{SUPPLEMENTARY MATERIAL}

Age-related vision loss is a leading cause of disability among aging adults and is particularly concerning given the increases in the US population's longevity. ${ }^{1,2}$ Given the economic burden associated with vision loss, estimated to be more than $\$ 3$ trillion globally, ${ }^{3}$ there are significant concerns about how vision loss impacts mental health and quality of life. Vision impairment has been associated with reduced quality of life, ${ }^{2}$ depression, ${ }^{4-6}$ and anxiety. ${ }^{7}$ Furthermore, the risks associated with vision loss and depression have increased risks of loneliness ${ }^{8}$ and suicide, ${ }^{9}$ even after adjusting for socioeconomic variables. ${ }^{10,11}$ This may also put individuals of lower economic status at a higher risk for visual issues and mental health difficulties. ${ }^{12}$ The functional implications of vision loss on mental health may be a different area for future research. As individuals may be more concerned about having to live with vision issues, their emotional distress is likely to increase and further interfere with activities of daily living (ADLs), jobs, athletics, or activities. Furthermore, the association of depression and visual impairment may be mediated by the individual's self-esteem and sense of mastery. ${ }^{13}$

There has also been recent literature that suggests the younger a person feels, the greater their sense of well-being, significant cognitive and physiological benefits, less inflammation, and increases in longevity. ${ }^{11}$ This also suggests that preventing visual issues may promote an individual's perception of themselves and play a significant role in promoting one's physiological, emotional, and cognitive functioning. Moreover, for individuals involved in athletics or activities as they age, reducing ocular issues could promote continued activity engagement and socialization, which has significant documented health benefits regarding mental health and preventing cognitive decline. ${ }^{14}$ Thus, addressing vision issues may reduce emotional distress, but the vital notion of preventing vision issues may promote optimal functioning.

\section{REFERENCES}

1. Bourne RRA, Flaxman SR, Braithwaite T, et al. Magnitude, temporal trends, and projections of the global prevalence of blindness and distance and near vision impairment: a systematic review and metaanalysis. Lancet Global Health 2017;5(9):e888-e897. doi:10.1016/ S2214109X(17)30293-03

2. Pelletier AL, Rojas-Roldan L, Coffin J. Vision loss in older adults. Am Fam Physician 2016;94(3):219-26

3. Gordois A, Cutler H, Pezzullo L, et al. An estimation of the worldwide economic and health burden of visual impairment. Glob Public Health 2012;7(5):465-481. doi:10.1080/ 17441692.2011 .6348155

4. Choi HG, Lee MJ, Lee S-M. Visual impairment and risk of depression: A longitudinal follow-up study using a national sample cohort. Sci Rep 2018;8:1. doi:10.1038/s41598-018-20374-5

5. Mayro EL, Murchison AP, Hark LA, et al. Prevalence of depressive symptoms and associated factors in an urban, ophthalmic population. Eur J Ophthalmol 2020;112067212090170. doi:10.1177/ 1120672120901701

6. Zhang X, Bullard KM, Cotch MF, et al. Association between depression and functional vision loss in persons 20 years of age or older in the United States, NHANES 2005-2008. JAMA Ophthalmol 2013;131(5):573.doi:10.1001jamaophthalmol.2013. 2597

7. Donoyama N, Takeda F. Mental health and related factors among massage practitioners with visual impairment. Ind Health. 2007;45(2):191-98. doi:10.2486/indhealth.45.191

8. Brunes A, Hansen B, Heir T. Loneliness among adults with visual impairment: prevalence, associated factors, and relationship to life satisfaction. Health Qual Life Outcomes 2019;17(1). doi:10.1186/ s12955-019-1096-y

9. Waern M. Burden of illness and suicide in elderly people: case-control study. BMJ 2002;324(7350):1355. doi:10.1136/ bmj.324.7350.13555

10. Rim TH, Lee CS, Lee SC, Chung B, Kim SS, Epidemiologic Survey Committee of the Korean Ophthalmological Society. Influence of visual acuity on suicidal ideation, suicide attempts and depression in South Korea. Br J Ophthalmol. 2015;99 (8):1112-1119. doi:10.1136/bjophthalmol-2014306518 
11. Wettstein M, Spuling SM, Cengia A, and Nowossadeck S. Feeling younger as a stress buffer: Subjective age moderates the effect of perceived stress on change in functional health. Psychology and Aging 2021;36(3), 322-337. https://doi.org/10.1037/pag0000608

12. Demmin DL, Silverstein SM. Visual impairment and mental health: unmet needs and treatment options. Clin Ophthalmol 2020;14:4229-4251. Published 2020 Dec 3. doi:10.2147/ OPTH.S258783
13. Maaswinkel IM, van der Aa HPA, van Rens GHMB, et al. Mastery and self-esteem mediate the association between visual acuity and mental health: a population-based longitudinal cohort study. BMC Psychiatr 2020;20:461. https:// doi.org/10.1186/s12888-020-02853-0

14. Brown CL, Robitaille A, Zelinski EM, Dixon RA, Hofer SM, Piccinin AM. Cognitive activity mediates the association between social activity and cognitive performance: A longitudinal study. Psychol Aging 2016;31(8):831-46. doi:10.1037/ pag0000134 